ALEA, Lat. Am. J. Probab. Math. Stat. 18, 249-263 (2021)

DOI: 10.30757/ALEA.v18-11

\title{
Moderate Deviation estimates for Nodal Lengths of Random Spherical Harmonics
}

\author{
Claudio Macci, Maurizia Rossi and Anna Paola Todino
}

\author{
Dipartimento di Matematica \\ Università degli Studi di Roma Tor Vergata \\ 1, Via della Ricerca Scientifica, 00133 Roma Italia. \\ E-mail address: macci@mat.uniroma2.it \\ URL: https://www.mat.uniroma2.it/ macci/ \\ Dipartimento di Matematica e Applicazioni \\ Università degli Studi di Milano-Bicocca \\ 55, Via Roberto Cozzi, 20125 Milano Italia. \\ E-mail address: maurizia.rossi@unimib.it \\ $U R L:$ https://www.unimib.it/maurizia-rossi \\ Fakultät für Mathematik \\ Ruhr-Universität Bochum \\ 150, Universitätstraße, 44801 Bochum Deutschland. \\ E-mail address: anna.todino@ruhr-uni-bochum.de \\ URL: https://www.ruhr-uni-bochum.de/ffm/Lehrstuehle/stochastik/todino.html
}

\begin{abstract}
We prove Moderate Deviation estimates for nodal lengths of random spherical harmonics both on the whole sphere and on shrinking spherical domains. Central Limit Theorems for the latter were recently established in Marinucci et al. (2020) and Todino (2020), respectively. Our proofs are based on the combination of a Moderate Deviation Principle by Schulte and Thäle (2016) for sequences of random variables living in a fixed Wiener chaos with a well-known result based on the concept of exponential equivalence
\end{abstract}

Received by the editors June 9th, 2020; accepted October 28th, 2020.

2010 Mathematics Subject Classification. 60F10, 60G15, 60G60.

Key words and phrases. Nodal length, Random Spherical Harmonics, Moderate Deviation Principles, Chaotic Expansions, Exponential Equivalence.

Research supported by MIUR Excellence Department Project awarded to the Department of Mathematics, University of Rome Tor Vergata CUP E83C18000100006 (C.M.), GNAMPAINdAM 2019 Project (C.M., M.R. and A.P.T.), ANR-17-CE40-0008 Project Unirandom (M.R.) and German Research Foundation (DFG) via RTG 2131 (A.P.T.). 


\section{Introduction: background and motivations}

1.1. Random spherical harmonics. Let $\mathbb{S}^{2}$ denote the two-dimensional unit sphere with the round metric and $\Delta$ the spherical Laplacian. In standard spherical coordinates $(\theta, \varphi) \in[0, \pi] \times[0,2 \pi)$, where $\theta$ is the colatitude, the metric takes the form

$$
d x^{2}=d \theta^{2}+\sin ^{2} \theta d \varphi^{2} .
$$

It is well-known that the spectrum of $\Delta$ is purely discrete, its eigenvalues are of the form $-\ell(\ell+1)$ where $\ell \in \mathbb{N}$ and, for each $\ell$, the family of the so-called spherical harmonics of degree $\ell\left\{Y_{\ell, m}, m=1, \ldots, 2 \ell+1\right\}$ is a real orthonormal basis of the $\ell$-th eigenspace (Marinucci and Peccati, 2011, §3.4).

Definition 1.1. For $\ell \in \mathbb{N}$, the $\ell$-th random spherical harmonic $T_{\ell}$ is a centered Gaussian field on $\mathbb{S}^{2}$ whose covariance kernel is given by

$$
\operatorname{Cov}\left(T_{\ell}(x), T_{\ell}(y)\right)=P_{\ell}(\cos d(x, y)), \quad x, y \in \mathbb{S}^{2},
$$

where $P_{\ell}$ denotes the Legendre polynomial (Marinucci and Peccati, 2011, §13.1.2) of degree $\ell$ and $d(x, y)$ the spherical geodesic distance (see (1.1)) between $x$ and $y$.

Equivalently, one can define $T_{\ell}$ as follows

$$
T_{\ell}(x):=\sqrt{\frac{4 \pi}{2 \ell+1}} \sum_{m=1}^{2 \ell+1} a_{\ell, m} Y_{\ell, m}(x), \quad x \in \mathbb{S}^{2},
$$

where $\left\{a_{\ell, m}, m=1, \ldots, 2 \ell+1\right\}$ are standard Gaussian and independent random variables, defined on a probability space $(\Omega, \mathcal{F}, \mathbb{P})$. Actually, from the addition formula (Marinucci and Peccati, 2011, (3.42)) for random spherical harmonics the covariance kernel of $T_{\ell}$ in (1.3) is given by (1.2). It is immediate that $T_{\ell}$ is isotropic, and that it is $\mathbb{P}$-a.s. an eigenfunction of the spherical Laplacian with eigenvalue $-\ell(\ell+1)$. We can assume $\left\{T_{\ell}, \ell \in \mathbb{N}\right\}$ to be independent random fields, indeed they are the Fourier components of isotropic Gaussian fields on the sphere, see e.g. Baldi et al. (2007) and Marinucci and Peccati $(2011, \S 5, \S 6)$.

Now we recall the Hilb's asymptotic formula: let $\epsilon>0$, uniformly for $\theta \in[0, \pi-\epsilon]$ and $\ell \in \mathbb{N}_{\geq 1}$

$$
P_{\ell}(\cos \theta)=\sqrt{\frac{\theta}{\sin \theta}} J_{0}((\ell+1 / 2) \theta)+O\left(\ell^{-3 / 2}\right),
$$

where $J_{0}$ is the Bessel function Szegó $(1975, \S 1.71)$ of the first kind of order zero, see the conventions below for the meaning of the $O$-notation. The scaling limit, as $\ell \rightarrow+\infty$, of $T_{\ell}$ is the so-called Berry's Random Wave model which, according to Berry's conjecture (Berry, 1977), should model the local behavior of high-energy deterministic eigenfunctions on "generic chaotic" surfaces.

Conventions. Given two sequences of positive real numbers $\left\{x_{n}, n \in \mathbb{N}\right\}$ and $\left\{y_{n}, n \in \mathbb{N}\right\}$ we will write $x_{n}=O\left(y_{n}\right)$ if the ratio $x_{n} / y_{n}$ is asymptotically bounded, and $x_{n}=o\left(y_{n}\right)$ if $\lim _{n \rightarrow+\infty} x_{n} / y_{n}=0$. Moreover, we will write $x_{n} \approx y_{n}$ if both $x_{n}=O\left(y_{n}\right)$ and $y_{n}=O\left(x_{n}\right)$ hold.

1.2. Nodal lengths: asymptotic distribution. Let us consider the nodal $\operatorname{set} T_{\ell}^{-1}(0):=$ $\left\{x \in \mathbb{S}^{2}: T_{\ell}(x)=0\right\}$. It is well-known that $T_{\ell}^{-1}(0)$ is an $\mathbb{P}$-a.s. smooth curve whose connected components are homeomorphic to the circle. We are interested in the 
high-energy geometry of the nodal set, in particular in the asymptotic behavior, as $\ell \rightarrow+\infty$, of the nodal length

$$
\mathcal{L}_{\ell}:=\operatorname{length}\left(T_{\ell}^{-1}(0)\right)
$$

The latter received great attention also in view of Yau's conjecture on nodal volumes of deterministic eigenfunctions on compact smooth Riemannian manifolds (Yau, 1982). We collect the main known results on the distribution of $\mathcal{L}_{\ell}$ in a single theorem (Theorem 1.2 below): the expected length was studied in Bérard (1985), the asymptotic variance in Wigman (2010) and the second order fluctuations of $\mathcal{L}_{\ell}$ in Marinucci et al. (2020). In order to state them, let us recall that, for integrable real random variables $X, Y$, the Wasserstein distance between $X$ and $Y$ is defined as $d_{W}(X, Y):=\sup _{h}|\mathbb{E}[h(X)]-\mathbb{E}[h(Y)]|$, where the supremum is taken over the set of Lipschitz functions whose Lipschitz constant is $\leq 1$. From now on, $Z \sim \mathcal{N}(0,1)$ will denote a standard Gaussian random variable.

Theorem 1.2. For every $\ell \in \mathbb{N}$

$$
\mathbb{E}\left[\mathcal{L}_{\ell}\right]=\frac{4 \pi}{2 \sqrt{2}} \sqrt{\ell(\ell+1)} .
$$

As $\ell \rightarrow+\infty$, we have

$$
\operatorname{Var}\left(\mathcal{L}_{\ell}\right)=\frac{1}{32} \log \ell(1+o(1))
$$

Moreover, denoting

$$
\widetilde{\mathcal{L}}_{\ell}:=\frac{\mathcal{L}_{\ell}-\mathbb{E}\left[\mathcal{L}_{\ell}\right]}{\sqrt{\operatorname{Var}\left(\mathcal{L}_{\ell}\right)}}
$$

a quantitative CLT in Wasserstein distance holds, i.e., as $\ell \rightarrow+\infty$

$$
d_{W}\left(\widetilde{\mathcal{L}}_{\ell}, Z\right)=O\left((\log \ell)^{-1 / 2}\right) .
$$

Note that, from (1.6) and (1.7) we have $\mathcal{L}_{\ell} / \sqrt{\ell(\ell+1)} \rightarrow 4 \pi /(2 \sqrt{2}) \mathbb{P}$-a.s. as $\ell \rightarrow+\infty$, consistently with Yau's conjecture Yau (1982). From (1.8) we conclude in particular that the asymptotic distribution of the nodal length is Gaussian. Moreover, from (1.8) and Nourdin and Peccati (2012, (C.2.6))

$$
d_{K o l}\left(\widetilde{\mathcal{L}}_{\ell}, Z\right)=O\left((\log \ell)^{-1 / 4}\right)
$$

where for arbitrary real random variables $X, Y$, the Kolmogorov distance between $X$ and $Y$ is defined as $d_{K o l}(X, Y):=\sup _{x \in \mathbb{R}}|\mathbb{P}(X \leq x)-\mathbb{P}(Y \leq x)|$.

1.2.1. Shrinking spherical domains. In Todino (2020) the asymptotic behavior of the nodal length in shrinking spherical domains was investigated. Fixed a point $x_{0} \in \mathbb{S}^{2}$, let $B_{r}$ denote the spherical cap of radius $r>0$ centered at $x_{0}$ and consider the length of nodal lines in $B_{r}$

$$
\mathcal{L}_{\ell, B_{r}}:=\operatorname{length}\left(T_{\ell}^{-1}(0) \cap B_{r}\right) .
$$

We recall that the area of $B_{r}$ is equal to $2 \pi(1-\cos r)$. We have the following results summarized in the next theorem.

Theorem 1.3. For any $r>0$

$$
\mathbb{E}\left[\mathcal{L}_{\ell, B_{r}}\right]=\frac{2 \pi(1-\cos r)}{2 \sqrt{2}} \sqrt{\ell(\ell+1)} .
$$


For a sequence of radii $\left\{r_{\ell}, \ell \in \mathbb{N}\right\}$ converging to zero not too rapidly $\left(r_{\ell} \ell \rightarrow+\infty\right)$ we have, as $\ell \rightarrow+\infty$,

$$
\operatorname{Var}\left(\mathcal{L}_{\ell, B_{r_{\ell}}}\right)=\frac{1}{256} r_{\ell}^{2} \log \left(r_{\ell} \ell\right)+O\left(r_{\ell}^{2}\right) .
$$

Moreover, denoting

$$
\widetilde{\mathcal{L}}_{\ell, B_{r_{\ell}}}:=\frac{\mathcal{L}_{\ell, B_{r_{\ell}}}-\mathbb{E}\left[\mathcal{L}_{\ell, B_{r_{\ell}}}\right]}{\sqrt{\operatorname{Var}\left(\mathcal{L}_{\ell, B_{r_{\ell}}}\right)}}
$$

we have a quantitative CLT in Wasserstein distance as $\ell \rightarrow+\infty$

$$
d_{W}\left(\widetilde{\mathcal{L}}_{\ell, B_{r_{\ell}}}, Z\right)=O\left(\left(\log r_{\ell} \ell\right)^{-1 / 2}\right) .
$$

Indeed (1.12) follows from the proof of Theorem 2.2 in Todino (2020) (at the end of §5.2.3) where in particular it is proved that the distribution of the nodal length in shrinking domains is asymptotically Gaussian. As in the previous case, we can also deduce that, as $\ell \rightarrow+\infty$,

$$
d_{K o l}\left(\widetilde{\mathcal{L}}_{\ell, B_{r_{\ell}}}, Z\right)=O\left(\left(\log r_{\ell} \ell\right)^{-1 / 4}\right) .
$$

In this paper we are interested in refinements of Central Limit Theorems stated above (Theorems 1.2 and 1.3).

1.3. Moderate Deviation Principles. The theory of Large Deviations allows an asymptotic computation of small probabilities at exponential scales. Here we start by recalling a basic definition. From now on let $(\Omega, \mathcal{F}, \mathbb{P})$ be a complete probability space, and $\left\{X_{n}, n \in \mathbb{N}\right\}$ a sequence of real-valued random variables: for each $n$ the map

$$
X_{n}: \Omega \rightarrow \mathbb{R}
$$

is measurable with respect to $\mathcal{F}$ and $\mathcal{B}(\mathbb{R})$ (the Borel $\sigma$-field of $\mathbb{R}$ ) on $\Omega$ and $\mathbb{R}$ respectively.

Definition 1.4. We say that $\left\{X_{n}, n \in \mathbb{N}\right\}$ satisfies the Large Deviation Principle (LDP) with speed $0 \leq s_{n} \nearrow+\infty$ and good rate function ${ }^{1} \mathcal{I}$ if for every $\alpha \geq 0$ the level set $\{x: \mathcal{I}(x) \leq \alpha\}$ is compact and for all $B \in \mathcal{B}(\mathbb{R})$ we have

$$
\begin{aligned}
-\inf _{x \in B} \mathcal{I}(x) & \leq \liminf _{n \rightarrow+\infty} \frac{1}{s_{n}} \log \mathbb{P}\left(X_{n} \in B\right) \\
& \leq \limsup _{n \rightarrow+\infty} \frac{1}{s_{n}} \log \mathbb{P}\left(X_{n} \in B\right) \leq-\inf _{x \in \bar{B}} \mathcal{I}(x),
\end{aligned}
$$

where $\stackrel{\circ}{B}$ (resp. $\bar{B}$ ) denotes the interior (resp. the closure) of $B$.

Let us now recall the notion of exponential equivalence (Dembo and Zeitouni, 1998, Definition 4.2.10) related to the question whether the LDP for $\left\{Y_{n}, n \in \mathbb{N}\right\}$ can be deduced from the LDP for $\left\{X_{n}, n \in \mathbb{N}\right\},\left\{Y_{n}, n \in \mathbb{N}\right\}$ being another sequence of real-valued random variables.

Definition 1.5. We say that $\left\{X_{n}, n \in \mathbb{N}\right\}$ and $\left\{Y_{n}, n \in \mathbb{N}\right\}$ are exponentially equivalent at speed $0 \leq s_{n} \nearrow+\infty$ if, for every $\delta>0$,

$$
\limsup _{n \rightarrow+\infty} \frac{1}{s_{n}} \log \mathbb{P}\left(\left|X_{n}-Y_{n}\right|>\delta\right)=-\infty
$$

\footnotetext{
${ }^{1}$ See $§ 1.2$ in Dembo and Zeitouni (1998) for details.
} 
As far as the LDP is concerned, exponentially equivalent sequences of random variables are indistinguishable (Dembo and Zeitouni, 1998, Theorem 4.2.13).

Lemma 1.6. Assume that $\left\{X_{n}, n \in \mathbb{N}\right\}$ satisfies the LDP with speed $s_{n}$ and good rate function $\mathcal{I}$. Then, if $\left\{X_{n}, n \in \mathbb{N}\right\}$ and $\left\{Y_{n}, n \in \mathbb{N}\right\}$ are exponentially equivalent at speed $s_{n}$, the same LDP holds for $\left\{Y_{n}, n \in \mathbb{N}\right\}$.

A Moderate Deviation Principle (MDP) is a class of LDPs for families of random variables depending on the choice of certain scalings in a suitable class. Moreover, all these LDPs (whose speed function depends on the scaling) are ruled by the same quadratic rate function vanishing at zero (actually, usually one deals with families of centered random variables, or asymptotically centered). In several cases the choice of the scaling parameters allows to fill the gap between the convergence in probability to a constant and the convergence in law to a centered Gaussian random variable.

Example 1.7. Let $\left\{Z_{n}, n \in \mathbb{N}\right\}$ be a sequence of i.i.d. real-valued random variables such that the moment-generating function

$$
\mathbb{R} \ni \lambda \mapsto \log \mathbb{E}\left[\mathrm{e}^{\lambda Z_{1}}\right]
$$

is finite in some ball around the origin, $\mathbb{E}\left[Z_{1}\right]=0$ and $\operatorname{Var}\left(Z_{1}\right)=1$. Let us define for each $n$ the random variable

$$
X_{n}:=\sum_{i=1}^{n} Z_{i} / \sqrt{n}
$$

For any sequence of positive numbers $\left\{a_{n}, n \in \mathbb{N}\right\}$ such that

$$
a_{n} \rightarrow+\infty, \quad a_{n} / \sqrt{n} \rightarrow 0,
$$

a MDP with speed $a_{n}^{2}$ and rate function $\mathcal{I}(x):=x^{2} / 2, x \in \mathbb{R}$, holds for $\left\{X_{n}, n \in \mathbb{N}\right\}$, namely for every Borel set $B \subset \mathbb{R}$

$$
\begin{aligned}
-\inf _{x \in \dot{B}} \mathcal{I}(x) & \leq \liminf _{n \rightarrow+\infty} \frac{1}{a_{n}^{2}} \log \mathbb{P}\left(X_{n} / a_{n} \in B\right) \\
& \leq \limsup _{n \rightarrow+\infty} \frac{1}{a_{n}^{2}} \log \mathbb{P}\left(X_{n} / a_{n} \in B\right) \leq-\inf _{x \in \bar{B}} \mathcal{I}(x),
\end{aligned}
$$

where $\stackrel{\circ}{B}$ (resp. $\bar{B}$ ) denotes the interior (resp. the closure) of $B$.

The classical example Dembo and Zeitouni (1998, Theorem 3.7.1) recalled just above concerns the empirical means of i.i.d. random variables; indeed, if these random variables have finite moment generating function in a neighborhood of the origin, a class of LDPs holds filling the gap between the asymptotic regimes of the Law of Large Numbers (LLN) and the Central Limit Theorem (CLT): indeed, using the same notation as in Example 1.7, as $n \rightarrow+\infty$ we have

$$
X_{n} / \sqrt{n} \rightarrow 0 \quad \mathbb{P}-\text { a.s. }, \quad X_{n} \stackrel{d}{\rightarrow} Z \sim \mathcal{N}(0,1),
$$

where $\rightarrow^{d}$ denotes convergence in distribution.

Furthermore, for completeness, we recall that a LDP linked to a LLN is provided by the celebrated Cramér Theorem (Dembo and Zeitouni, 1998, Theorem 2.2.3). 


\section{Main results and outline of the paper}

2.1. Statement of main results. Our main results concern MDPs which refine Theorems 1.2 and 1.3, namely a class of LDPs for nodal lengths of random spherical harmonics for certain scalings $\left\{a_{\ell}, \ell \in \mathbb{N}\right\}$ that tend to infinity slowly (see conditions (2.1) and (2.2) in Theorems 2.1 and 2.2 below), with speed $a_{\ell}^{2}$, and common quadratic rate function $\mathcal{I}(x)=x^{2} / 2, x \in \mathbb{R}$. Moreover, for $a_{\ell}=1$ (and in such a case the condition $a_{\ell} \rightarrow+\infty$ in (2.1) and (2.2) fails) we have the convergence in law to the standard Normal distribution (Theorem 1.2 and Theorem 1.3). Recall the preliminaries in Section 1.3 and the discussion just after the statement of Lemma 1.6.

Theorem 2.1. Let $\left\{a_{\ell}, \ell \in \mathbb{N}\right\}$ be any sequence of positive numbers such that, as $\ell \rightarrow+\infty$,

$$
a_{\ell} \rightarrow+\infty, \quad a_{\ell} / \sqrt{\log \log \ell} \rightarrow 0 .
$$

The sequence of random variables

$$
\left\{\widetilde{\mathcal{L}}_{\ell} / a_{\ell}, \ell \in \mathbb{N}\right\}
$$

satisfies a MDP with speed $a_{\ell}^{2}$ and rate function $\mathcal{I}(x)=x^{2} / 2, x \in \mathbb{R}$.

From now on, $\left\{r_{\ell}, \ell \in \mathbb{N}\right\}$ is a sequence of radii such that (see Theorem 1.3)

$$
r_{\ell} \ell \rightarrow+\infty \text {. }
$$

Theorem 2.2. Let $\left\{a_{\ell}, \ell \in \mathbb{N}\right\}$ be any sequence of positive numbers such that, as $\ell \rightarrow+\infty$,

$$
a_{\ell} \rightarrow+\infty, \quad a_{\ell} / \sqrt{\log \log r_{\ell} \ell} \rightarrow 0 .
$$

The sequence of random variables

$$
\left\{\widetilde{\mathcal{L}}_{\ell, B_{r_{\ell}}} / a_{\ell}, \ell \in \mathbb{N}\right\}
$$

satisfies a MDP with speed $a_{\ell}^{2}$ and rate function $\mathcal{I}(x)=x^{2} / 2, x \in \mathbb{R}$.

To the best of our knowledge, Theorem 2.1 and Theorem 2.2 are the first MD estimates for Lipschitz-Killing curvatures of excursion sets of Laplacian Gaussian eigenfunctions on manifolds and on shrinking domains on manifolds, respectively.

Remark 2.3 (Lipschitz-Killing curvatures). The conditions $a_{\ell} / \sqrt{\log \log \ell} \rightarrow 0$ in (2.1) and $a_{\ell} / \sqrt{\log \log r_{\ell} \ell} \rightarrow 0$ in (2.2) are plausibly not optimal; this is a drawback of the proof technique we decided to adopt. Our choice was based on the fact that we do not have any information on the moment-generating function of the nodal length and, more importantly, on the shortness of our argument as well as on its flexibility. Indeed, our strategy can be immediately adapted to prove MD estimates for the other Lipschitz-Killing (LK) curvatures of excursion sets at any level of random spherical harmonics such as the length of level curves, the excursion area and the Euler-Poincaré characteristic (see e.g. Cammarota and Marinucci, 2018; Marinucci and Wigman, 2014; Todino, 2019 and the references therein). Actually, our approach works well independently of the underlying manifold as long as the random model is Gaussian and long-memory, for instance in the case of arithmetic random waves, see Benatar et al. (2020); Cammarota (2019); Marinucci et al. (2016); Nourdin et al. (2019); Peccati and Rossi (2018) and the references therein. 
2.2. On the proofs of the main results. The nodal length $\mathcal{L}_{\ell}$ in (1.5) is a finitevariance functional of the Gaussian field $T_{\ell}$ (Definition 1.1) hence it can be written as an orthogonal series, the so-called Wiener-Itto chaos expansion, converging in $L^{2}(\mathbb{P})$ of the form

$$
\mathcal{L}_{\ell}=\mathbb{E}\left[\mathcal{L}_{\ell}\right]+\sum_{q=1}^{+\infty} \mathcal{L}_{\ell}[q],
$$

where $\mathcal{L}_{\ell}[q]$ is the orthogonal projection of $\mathcal{L}_{\ell}$ onto the so-called Wiener chaos of order $q$. The expansion in (2.3) relies on the fact that the family of suitably normalized Hermite polynomials $\left\{H_{q}, q \in \mathbb{N}\right\}$ is an orthonormal basis for the space of square integrable functions on the real line with respect to the Gaussian density. Recall that $H_{0} \equiv 1$ and

$$
H_{q}(t):=(-1)^{q} \phi^{-1}(t) \frac{d^{q}}{d t^{q}} \phi(t), \quad t \in \mathbb{R}, q \in \mathbb{N}_{\geq 1},
$$

where $\phi$ denotes the standard Gaussian density. In particular $H_{1}(t)=1, H_{2}(t)=$ $t^{2}-1, H_{3}(t)=t^{3}-3 t$ and $H_{4}(t)=3 t^{4}-6 t^{2}+3$. It turns out that $\mathcal{L}_{\ell}-\mathbb{E}\left[\mathcal{L}_{\ell}\right]$ is asymptotically equivalent, in the $L^{2}(\mathbb{P})$-sense, to its fourth chaotic component $\mathcal{L}_{\ell}[4]$ which is moreover fully correlated, as $\ell \rightarrow+\infty$, to the sample trispectrum of $T_{\ell}$. More precisely let us define, for $\ell \in \mathbb{N}$,

$$
\mathcal{M}_{\ell}:=-\frac{1}{4} \sqrt{\frac{\ell(\ell+1)}{2}} \frac{1}{4 !} \int_{\mathbb{S}^{2}} H_{4}\left(T_{\ell}(x)\right) d x ;
$$

Theorem 1.2 in Marinucci et al. (2020) states that, as $\ell \rightarrow+\infty$,

$$
\mathbb{E}\left[\left|\widetilde{\mathcal{L}}_{\ell}-\widetilde{\mathcal{M}}_{\ell}\right|^{2}\right]=O\left((\log \ell)^{-1}\right)
$$

where

$$
\widetilde{\mathcal{M}}_{\ell}:=\frac{\mathcal{M}_{\ell}}{\sqrt{\operatorname{Var}\left(\mathcal{M}_{\ell}\right)}} .
$$

Now recall that the sequence of random variables $\left\{\widetilde{\mathcal{M}}_{\ell}, \ell \in \mathbb{N}\right\}$ lives in the fourth Wiener chaos. On that space, convergence in law to a standard Gaussian random variable can be proved Nualart and Peccati (2005); Nourdin and Peccati (2009) showing that its fourth cumulant goes to zero, hence

$$
\operatorname{Cum}_{4}\left(\widetilde{\mathcal{M}}_{\ell}\right) \rightarrow 0
$$

Theorem 1 in Schulte and Thäle (2016) ensures that under (2.7) a MDP holds for the normalized sample trispectrum. See also Döring and Eichelsbacher (2013) for MDP via cumulants and Ledoux (1990) for LDP for Wiener chaos.

In this paper, as a preliminary result (Lemma 4.1), firstly we will establish a $\operatorname{MDP}$ for $\left\{\widetilde{\mathcal{M}}_{\ell} / a_{\ell}, \ell \in \mathbb{N}\right\}$ with the rate function $\mathcal{I}$ in Theorem 1.2 and speed $a_{\ell}^{2}$, whenever as $\ell \rightarrow+\infty$

$$
a_{\ell} \rightarrow+\infty, \quad a_{\ell} /(\log \ell)^{1 / 7} \rightarrow 0 .
$$

Then, in order to deduce a MDP for the whole series on the right hand side of (2.3), i.e. to establish Theorem 2.1, we will prove that $\left\{\widetilde{\mathcal{M}}_{\ell} / a_{\ell}, \ell \in \mathbb{N}\right\}$ and $\left\{\widetilde{\mathcal{L}}_{\ell} / a_{\ell}, \ell \in \mathbb{N}\right\}$ are exponentially equivalent (Definition 1.5) at speed $a_{\ell}^{2}$ provided that $a_{\ell}$ goes to infinity sufficiently slowly (see (2.1)) according to (2.6). Finally Lemma 1.6 will allow to conclude. 
The proof of Theorem 2.2 relies on the same ideas as those developed just before for the proof of Theorem 2.1.

2.3. Plan of the paper. In $\S 3$ we recall both the notion of Wiener chaos and the chaotic expansion (2.3) for the nodal length of random spherical harmonics. In particular, in $\S 3.3$ we recall the reduction principle for nodal lengths on the sphere and on shrinking domains leading in particular to (2.6). Finally in $\S 4$ we give the proofs of our main results.

\section{Nodal lengths and Wiener chaos}

3.1. Wiener chaos. It is well-known (Nourdin and Peccati, 2012, Proposition 1.4.2) that the family $\left\{H_{q} / \sqrt{q !}, q \in \mathbb{N}\right\}$ of suitably normalized Hermite polynomails (2.4) is a complete orthonormal system in the space of square integrable real functions $L^{2}(\phi)$ with respect to the standard Gaussian measure on the real line.

Random spherical harmonics (1.3) are linear combinations of i.i.d. standard Gaussian random variables $\left\{a_{\ell, m}: \ell=1,2, \ldots, m=1, \ldots, 2 \ell+1\right\}$; we define accordingly the space $X$ to be the closure in $L^{2}(\mathbb{P})$ of $\operatorname{lin}\left\{a_{\ell, m}: \ell=1,2, \ldots, m=\right.$ $1, \ldots, 2 \ell+1\}$, thus $X$ is a real centered Gaussian Hilbert subspace of $L^{2}(\mathbb{P})$. Now let $q \in \mathbb{N}$; the $q$-th Wiener chaos $C_{q}$ associated with $X$ is defined as the closure in $L^{2}(\mathbb{P})$ of all real finite linear combinations of random variables of the form

$$
H_{p_{1}}\left(x_{1}\right) H_{p_{2}}\left(x_{2}\right) \cdots H_{p_{k}}\left(x_{k}\right)
$$

for $k \in \mathbb{N}_{\geq 1}$, where $p_{1}, \ldots, p_{k} \in \mathbb{N}$ satisfy $p_{1}+\cdots+p_{k}=q$, and $\left(x_{1}, x_{2}, \ldots, x_{k}\right)$ is a standard Gaussian vector extracted from $X$ (plainly, $C_{0}=\mathbb{R}$ and $C_{1}=X$ ). Note that (from (1.3)) for every $\ell$ the random fields $T_{\ell}$ and $\nabla T_{\ell}$ viewed as collections of Gaussian random variables indexed by $x \in \mathbb{S}^{2}$ are all lying in $X$.

Taking into account the orthonormality and completeness of $\left\{H_{q} / \sqrt{q !}, q \in \mathbb{N}\right\}$ in $L^{2}(\phi)$, together with a monotone class argument (see e.g. Nourdin and Peccati, 2012, Theorem 2.2.4), one can prove that $C_{q} \perp C_{q^{\prime}}$ in $L^{2}(\mathbb{P})$ whenever $q \neq q^{\prime}$, and moreover

$$
L_{X}^{2}(\mathbb{P})=\bigoplus_{q=0}^{\infty} C_{q},
$$

where $L_{X}^{2}(\mathbb{P}):=L^{2}(\Omega, \sigma(X), \mathbb{P})$, that is, every finite-variance real-valued functional $F$ of $X$ admits a unique representation as a series, converging in $L_{X}^{2}(\mathbb{P})$, of the form

$$
F=\sum_{q=0}^{\infty} F[q],
$$

$F[q]:=\operatorname{proj}\left(F \mid C_{q}\right)$ being the orthogonal projection of $F$ onto $C_{q}$ (in particular, $F[0]=\mathbb{E}[F]$ ). For a complete discussion on Wiener chaos see Nourdin and Peccati $(2012, \S 2.2)$ and the references therein.

3.2. Nodal length: chaos expansion. Let $B \subseteq \mathbb{S}^{2}$ be a "nice" subset of the sphere. For our purpose it suffices to take $B$ as the whole sphere or a spherical cap. The nodal length $\mathcal{L}_{\ell, B}:=\operatorname{length}\left(T_{\ell}^{-1}(0) \cap B\right)$ in $B$ at frequency $\ell$ (plainly, $\mathcal{L}_{\ell, \mathbb{S}^{2}} \equiv \mathcal{L}_{\ell}$ ) can be formally written as

$$
\mathcal{L}_{\ell, B}=\int_{B} \delta_{0}\left(T_{\ell}(x)\right)\left\|\nabla T_{\ell}(x)\right\| d x
$$


where $\delta_{0}$ stands for the Dirac mass in $0, \nabla T_{\ell}$ is the gradient field and $\|\cdot\|$ denotes the Euclidean norm in $\mathbb{R}^{2}$. Indeed, let us consider the $\epsilon$-approximating random variable

$$
\mathcal{L}_{\ell, B}^{\epsilon}:=\frac{1}{2 \epsilon} \int_{B} \chi_{[-\epsilon, \epsilon]}\left(T_{\ell}(x)\right)\left\|\nabla T_{\ell}(x)\right\| d x
$$

where $0<\epsilon<1$ and $\chi_{[-\epsilon, \epsilon]}$ denotes the indicator function of the interval $[-\epsilon, \epsilon]$. It is possible to prove that

$$
\lim _{\epsilon \rightarrow 0} \mathcal{L}_{\ell, B}^{\epsilon}=\operatorname{length}\left(T_{\ell}^{-1}(0) \cap B\right)
$$

both $\mathbb{P}-$ a.s. and in $L^{2}(\mathbb{P})$, see Marinucci et al. (2020); Nourdin et al. (2019), thus justifying (3.2). In particular, $\mathcal{L}_{\ell, B} \in L_{X}^{2}(\mathbb{P})$. The integral representation (3.2) can be equivalently written as

$$
\mathcal{L}_{\ell, B}=\sqrt{\frac{\ell(\ell+1)}{2}} \int_{B} \delta_{0}\left(T_{\ell}(x)\right)\left\|\widetilde{\nabla} T_{\ell}(x)\right\| d x,
$$

where $\widetilde{\nabla}$ is the normalized gradient, i.e. $\widetilde{\nabla}:=\nabla / \sqrt{\frac{2}{\ell(\ell+1)}}$ (thus pointwise the components of the normalized gradient have unit variance, see §3.2.1 in Marinucci et al., 2020 for details). Let us now recall the chaotic expansion (3.1) for $\mathcal{L}_{\ell, B}$

$$
\mathcal{L}_{\ell, B}=\sum_{q=0}^{+\infty} \mathcal{L}_{\ell, B}[2 q],
$$

where $\mathcal{L}_{\ell, B}[2 q]$ denotes the orthogonal projection of $\mathcal{L}_{\ell, B}$ onto $C_{2 q}$. (Note that projections on odd chaoses vanish since the integrand functions in (3.3) are both even.) In Marinucci et al. $(2020, \S 2)$ the terms of the series on the right hand side of (3.4) are explicitly given (see also Marinucci et al., 2016). Let us introduce the two sequences of real numbers $\left\{\beta_{2 k}\right\}_{k=0}^{+\infty}$ and $\left\{\alpha_{2 n, 2 m}\right\}_{n, m=0}^{+\infty}$ corresponding to the (formal) chaotic coefficients of the Dirac mass at 0 and the Euclidean norm respectively: for $k, n, m \in \mathbb{N}$

$$
\beta_{2 k}:=\frac{1}{\sqrt{2 \pi}} H_{2 k}(0), \quad \alpha_{2 n, 2 m}:=\sqrt{\frac{\pi}{2}} \frac{(2 n) !(2 m) !}{n ! m !} \frac{1}{2^{n+m}} p_{n+m}\left(\frac{1}{4}\right),
$$

where $p_{N}$ is the swinging factorial coefficient

$$
p_{N}(x):=\sum_{j=0}^{N}(-1)^{N+j}\left(\begin{array}{c}
N \\
j
\end{array}\right) \frac{(2 j+1) !}{(j !)^{2}} x^{j}
$$

for $x \in \mathbb{R}$. The $2 q$-th chaotic projection of the nodal length restricted to $B$ is

$$
\begin{aligned}
\mathcal{L}_{\ell, B}[2 q]= & \sqrt{\frac{\ell(\ell+1)}{2}} \sum_{u=0}^{q} \sum_{k=0}^{u} \frac{\alpha_{2 k, 2 u-2 k} \beta_{2 q-2 u}}{(2 k) !(2 u-2 k) !(2 q-2 u) !} \\
& \times \int_{B} H_{2 q-2 u}\left(T_{\ell}(x)\right) H_{2 k}\left(\widetilde{\partial}_{1 ; x} T_{\ell}(x)\right) H_{2 u-2 k}\left(\widetilde{\partial}_{2 ; x} T_{\ell}(x)\right) d x,
\end{aligned}
$$

where we use spherical coordinates (colatitude $\theta$, longitude $\varphi$ ) and for $x=\left(\theta_{x}, \varphi_{x}\right)$ we are using the notation

$$
\widetilde{\partial}_{1 ; x}=\left.(\ell(\ell+1) / 2)^{-1 / 2} \cdot \frac{\partial}{\partial \theta}\right|_{\theta=\theta_{x}}, \quad \widetilde{\partial}_{2 ; x}=\left.(\ell(\ell+1) / 2)^{-1 / 2} \cdot \frac{1}{\sin \theta} \frac{\partial}{\partial \varphi}\right|_{\theta=\theta_{x}, \varphi=\varphi_{x}} .
$$

Obviously $\mathcal{L}_{\ell, B}[0]=\frac{\operatorname{area}(B)}{2 \sqrt{2}} \sqrt{\ell(\ell+1)}=\mathbb{E}\left[\mathcal{L}_{\ell, B}\right]$. 
3.3. Nodal lengths: reduction principles.

3.3.1. On the sphere. From (3.5) for $q=1$, an application of Green's formula yields (see Marinucci and Rossi, 2020 and the references therein)

$$
\mathcal{L}_{\ell}[2]=0 .
$$

Let us consider, as in (2.5), the sample trispectrum

$$
\mathcal{M}_{\ell}:=-\frac{1}{4} \sqrt{\frac{\ell(\ell+1)}{2}} \frac{1}{4 !} \int_{\mathbb{S}^{2}} H_{4}\left(T_{\ell}(x)\right) d x ;
$$

from the properties of Hermite polynomials recalled in $\S 3.1$ and by (1.4) we have

$$
\mathbb{E}\left[\mathcal{M}_{\ell}\right]=0, \quad \operatorname{Var}\left(\mathcal{M}_{\ell}\right)=\frac{1}{32} \log \ell+O(1), \text { as } \ell \rightarrow+\infty
$$

(see Marinucci and Wigman, 2014, Lemma 3.2), cf. (1.7). Moreover, in Marinucci et al. (2020) it has been shown that the fourth chaotic projection $\mathcal{L}_{\ell}[4]$ (see (3.5) for $q=2$ ) is asymptotically (as $\ell \rightarrow+\infty$ ) equivalent, in the $L^{2}\left(\mathbb{P}\right.$ )-sense, to $\mathcal{M}_{\ell}$, and that the tail $\sum_{q \geq 3} \mathcal{L}_{\ell}[2 q]$ of the chaotic series (3.4) is negligible. To be more precise,

$$
\mathbb{E}\left[\left|\widetilde{\mathcal{L}}_{\ell}-\widetilde{\mathcal{M}}_{\ell}\right|^{2}\right]=O\left((\log \ell)^{-1}\right), \quad \text { as } \ell \rightarrow+\infty,
$$

which is (2.6). An application of the Fourth Moment Theorem (Nualart and Peccati, 2005; Nourdin and Peccati, 2009) gives (Marinucci and Wigman, 2014)

$$
d_{W}\left(\widetilde{\mathcal{M}}_{\ell}, Z\right) \leq \sqrt{\operatorname{Cum}_{4}\left(\widetilde{\mathcal{M}}_{\ell}\right)}=O\left((\log \ell)^{-1}\right), \quad \text { as } \ell \rightarrow+\infty,
$$

that together with the estimate (3.8) proves (1.8).

3.3.2. On shrinking spherical domains. Let us define the local sample trispectrum as

$$
\mathcal{M}_{\ell, B_{r_{\ell}}}:=-\frac{1}{4} \sqrt{\frac{\ell(\ell+1)}{2}} \frac{1}{4 !} \int_{B_{r_{\ell}}} H_{4}\left(T_{\ell}(x)\right) d x .
$$

It has zero mean and variance (recall that $r_{\ell} \ell \rightarrow+\infty$ ) given by

$$
\operatorname{Var}\left(\mathcal{M}_{\ell, B_{r_{\ell}}}\right)=\frac{1}{256} r_{\ell}^{2} \log r_{\ell} \ell+O\left(r_{\ell}^{2}\right)
$$

(see Todino, 2020, Proposition 3.4). In Todino (2020, Proposition 3.3) the asymptotic full correlation between the local nodal length $\mathcal{L}_{\ell, B_{r_{\ell}}}$ and the local sample trispectrum has been established and, in view of the orthogonality of the projections, this entails that $\mathcal{M}_{\ell, B_{r_{\ell}}}$ is the leading term of the chaos expansion of $\mathcal{L}_{\ell, B_{r_{\ell}}}$ in (3.5). Indeed, all the other projections are proved to be $O\left(r_{\ell}^{2}\right)$ in the $L^{2}$-sense (see the supplement article to Todino (2020), Appendix C) and hence we can conclude that

$$
\mathbb{E}\left[\left|\widetilde{\mathcal{L}}_{\ell, B_{r_{\ell}}}-\widetilde{\mathcal{M}}_{\ell, B_{r_{\ell}}}\right|^{2}\right]=O\left(\left(\log r_{\ell} \ell\right)^{-1}\right) .
$$

Hence the Fourth Moment Theorem (Nualart and Peccati, 2005; Nourdin and Peccati, 2009) gives (Todino, 2020, Lemma 5.4)

$$
d_{W}\left(\widetilde{\mathcal{M}}_{\ell, B_{r_{\ell}}}, Z\right) \leq \sqrt{\operatorname{Cum}_{4}\left(\widetilde{\mathcal{M}}_{\ell, B_{r_{\ell}}}\right)}=O\left(\left(\log r_{\ell} \ell\right)^{-1 / 2}\right)
$$

that together with the estimate (3.11) proves (1.12). 


\section{Proofs of the main results}

4.1. Proof of Theorem 2.1. Bearing in mind §3.3.1 we start with an auxiliary lemma of independent interest which provides the MDP for the sample trispectrum on the sphere.

Lemma 4.1. Let $\left\{a_{\ell}, \ell \in \mathbb{N}\right\}$ be any sequence of positive numbers such that, as $\ell \rightarrow+\infty$,

$$
a_{\ell} \rightarrow+\infty, \quad a_{\ell} /(\log \ell)^{1 / 7} \rightarrow 0 .
$$

Then the sequence of random variables $\left\{\widetilde{\mathcal{M}}_{\ell} / a_{\ell}, \ell \in \mathbb{N}\right\}$ satisfies a MDP with speed $a_{\ell}^{2}$ and rate function $\mathcal{I}(x)=x^{2} / 2, x \in \mathbb{R}$.

Proof: The random variable $\mathcal{M}_{\ell}$ belongs to the fourth Wiener chaos $C_{4}$ for each $\ell \in \mathbb{N}$. Recalling (3.7), from Marinucci and Wigman (2014, Lemma 3.3), we have that, as $\ell \rightarrow+\infty, \operatorname{Cum}_{4}\left(\mathcal{M}_{\ell}\right) \approx 1$. Hence we have, as $\ell \rightarrow+\infty$,

$$
\operatorname{Cum}_{4}\left(\widetilde{\mathcal{M}}_{\ell}\right)=\frac{\operatorname{Cum}_{4}\left(\mathcal{M}_{\ell}\right)}{\operatorname{Var}\left(\mathcal{M}_{\ell}\right)^{2}} \approx \frac{1}{\log ^{2} \ell}
$$

Now let $\left\{a_{\ell}, \ell \in \mathbb{N}\right\}$ be a positive sequence such that, as $\ell \rightarrow+\infty$,

$$
a_{\ell} \rightarrow+\infty, \quad a_{\ell} /\left(\Delta_{\ell}\right)^{1 / 3} \rightarrow 0,
$$

where

$$
\Delta_{\ell}:=\left(\sqrt{\operatorname{Cum}_{4}\left(\widetilde{\mathcal{M}}_{\ell}\right)}\right)^{-3 / 7} \approx(\log \ell)^{3 / 7}
$$

Note that (4.3) is equivalent to (4.1). Corollary 2 in Schulte and Thäle (2016) ensures that the sequence $\widetilde{\mathcal{M}}_{\ell} / a_{\ell}$ satisfies a MDP with speed $a_{\ell}^{2}$ and rate function $\mathcal{I}(x)=x^{2} / 2, x \in \mathbb{R}$.

Remark 4.2. Our argument leads to further results, namely expansions à la CramérPetrov (Rudzkis et al., 1978) for the sample trispectrum. We state here the result in a simplified form taken from Theorem 5 (i) in Schulte and Thäle (2016): there exist universal constants $c_{0}, c_{1}, c_{2}>0$ such that for $\Delta_{\ell} \geq c_{0}, 0 \leq x \leq c_{1} \Delta_{\ell}^{1 / 3}\left(\Delta_{\ell}\right.$ is defined as in (4.4)) it holds that

$$
\left|\log \frac{\mathbb{P}\left(\widetilde{\mathcal{M}}_{\ell} \geq x\right)}{1-\Phi(x)}\right| \leq c_{2} \frac{1+x^{3}}{\Delta_{\ell}^{1 / 3}}, \quad\left|\log \frac{\mathbb{P}\left(\widetilde{\mathcal{M}}_{\ell} \leq-x\right)}{\Phi(-x)}\right| \leq c_{2} \frac{1+x^{3}}{\Delta_{\ell}^{1 / 3}},
$$

where $\Phi$ denotes the standard Gaussian cumulative distribution function. An anonymous referee raised the interesting question whether expansions à la CramérPetrov hold for nodal lengths on the sphere and on shrinking spherical domains. We leave this as a topic for future research.

Proof of Theorem 2.1: We want to combine Lemma 1.6 and Lemma 4.1 for every speed $a_{\ell}$ satisfying (2.1); note that $a_{\ell} / \sqrt{\log \log \ell} \rightarrow 0$ implies $a_{\ell} /(\log \ell)^{1 / 7} \rightarrow 0$ (see (4.1)). In order to do so, we have to check that for every $\delta>0$

$$
\limsup _{\ell \rightarrow+\infty} \frac{1}{a_{\ell}^{2}} \log \mathbb{P}\left(a_{\ell}^{-1}\left|\widetilde{\mathcal{L}}_{\ell}-\widetilde{\mathcal{M}}_{\ell}\right|>\delta\right)=-\infty
$$


i.e., that the sequences of random variables $\left\{\widetilde{\mathcal{L}}_{\ell} / a_{\ell}, \ell \in \mathbb{N}\right\}$ and $\left\{\widetilde{\mathcal{M}}_{\ell} / a_{\ell}, \ell \in \mathbb{N}\right\}$ are exponentially equivalent (Definition 1.5) at speed $a_{\ell}^{2}$. Thanks to Markov inequality we have

$$
\mathbb{P}\left(a_{\ell}^{-1}\left|\widetilde{\mathcal{L}}_{\ell}-\widetilde{\mathcal{M}}_{\ell}\right|>\delta\right) \leq \frac{a_{\ell}^{-2} \mathbb{E}\left[\left|\widetilde{\mathcal{L}}_{\ell}-\widetilde{\mathcal{M}}_{\ell}\right|^{2}\right]}{\delta^{2}}
$$

so that

$$
\begin{aligned}
\limsup _{\ell \rightarrow+\infty} a_{\ell}^{-2} \log \mathbb{P}\left(a_{\ell}^{-1}\left|\widetilde{\mathcal{L}}_{\ell}-\widetilde{\mathcal{M}}_{\ell}\right|>\delta\right) & \leq \limsup _{\ell \rightarrow+\infty} a_{\ell}^{-2} \log \frac{a_{\ell}^{-2} \mathbb{E}\left[\left|\widetilde{\mathcal{L}}_{\ell}-\widetilde{\mathcal{M}}_{\ell}\right|^{2}\right]}{\delta^{2}} \\
& =\limsup _{\ell \rightarrow+\infty} a_{\ell}^{-2} \log \mathbb{E}\left[\left|\widetilde{\mathcal{L}}_{\ell}-\widetilde{\mathcal{M}}_{\ell}\right|^{2}\right] .
\end{aligned}
$$

Plugging (2.6) into (4.6) we get (4.5) whenever $a_{\ell}=o(\sqrt{\log \log \ell})$ as in (2.1).

4.2. Proof of Theorem 2.2. Here we refer to $§ 3.3 .2$ and we follow the same lines of the proof of Theorem 2.1. We first prove the following lemma which is of independent interest.

Lemma 4.3. Let $\left\{a_{\ell}, \ell \in \mathbb{N}\right\}$ be any sequence of positive numbers such that, as $\ell \rightarrow+\infty$,

$$
a_{\ell} \rightarrow+\infty, \quad a_{\ell} /\left(\log r_{\ell} \ell\right)^{1 / 14} \rightarrow 0 .
$$

Then the sequence of random variables $\left\{\widetilde{\mathcal{M}}_{\ell, B_{r_{\ell}}} / a_{\ell}, \ell \in \mathbb{N}\right\}$ satisfies a MDP with speed $a_{\ell}^{2}$ and rate function $\mathcal{I}(x)=x^{2} / 2, x \in \mathbb{R}$.

Proof: The random variable $\mathcal{M}_{\ell, B_{r_{\ell}}}$ in (3.9) belongs to the fourth Wiener chaos for each $\ell \in \mathbb{N}$. Recalling (3.10) and that from Todino (2020, Lemma 5.4), as $\ell \rightarrow+\infty$, $\operatorname{Cum}_{4}\left(\mathcal{M}_{\ell, B_{r_{\ell}}}\right)=O\left(r_{\ell}^{4} \log r_{\ell} \ell\right)$, we have

$$
\operatorname{Cum}_{4}\left(\widetilde{\mathcal{M}}_{\ell, B_{r_{\ell}}}\right)=\frac{\operatorname{Cum}_{4}\left(\mathcal{M}_{\ell, B_{r_{\ell}}}\right)}{\operatorname{Var}\left(\mathcal{M}_{\ell, B_{r_{\ell}}}\right)^{2}}=O\left(\frac{1}{\log r_{\ell} \ell}\right) .
$$

Now let $\left\{a_{\ell}, \ell \in \mathbb{N}\right\}$ be a positive sequence such that, as $\ell \rightarrow+\infty$,

$$
a_{\ell} \rightarrow+\infty, \quad a_{\ell} /\left(\Delta_{\ell, r_{\ell}}\right)^{1 / 3} \rightarrow 0,
$$

where

$$
\Delta_{\ell, r_{\ell}}^{-1}:=\left(\sqrt{\operatorname{Cum}_{4}\left(\widetilde{\mathcal{M}}_{\ell, B_{r_{\ell}}}\right)}\right)^{3 / 7}=O\left(\left(\log r_{\ell} \ell\right)^{-3 / 14}\right), \quad \text { as } \ell \rightarrow+\infty .
$$

Note that (4.7) implies (4.9). It follows that, as in the proof of Lemma 4.1, Corollary 2 in Schulte and Thäle (2016) ensures that the sequence $\left\{\widetilde{\mathcal{M}}_{\ell, B_{r_{\ell}}} / a_{\ell}, \ell \in \mathbb{N}\right\}$ satisfies a MDP with speed $a_{\ell}^{2}$ and rate function $\mathcal{I}(x)=x^{2} / 2$.

Analogous results as those in Remark 4.2 hold for the sample trispectrum restricted to a shrinking ball.

Proof of Theorem 2.2: Along the same lines of the proof of Theorem 2.1 we want to combine Lemma 1.6 and Lemma 4.3 for every speed $a_{\ell}$ satisfying (2.2); note that 
$a_{\ell} / \sqrt{\log \log r_{\ell} \ell} \rightarrow 0$ implies $a_{\ell} /\left(\log r_{\ell} \ell\right)^{1 / 14} \rightarrow 0$ (see (4.7)). To this aim, we have to check that for every $\delta>0$

$$
\limsup _{\ell \rightarrow+\infty} \frac{1}{a_{\ell}^{2}} \log \mathbb{P}\left(a_{\ell}^{-1}\left|\widetilde{\mathcal{L}}_{\ell, B_{r_{\ell}}}-\widetilde{\mathcal{M}}_{\ell, B_{r_{\ell}}}\right|>\delta\right)=-\infty
$$

Applying Markov inequality we have

$$
\mathbb{P}\left(a_{\ell}^{-1}\left|\widetilde{\mathcal{L}}_{\ell, B_{r_{\ell}}}-\widetilde{\mathcal{M}}_{\ell, B_{r_{\ell}}}\right|>\delta\right) \leq \frac{a_{\ell}^{-2} \mathbb{E}\left[\left|\widetilde{\mathcal{L}}_{\ell, B_{r_{\ell}}}-\widetilde{\mathcal{M}}_{\ell, B_{r_{\ell}}}\right|^{2}\right]}{\delta^{2}}
$$

and hence

$$
\begin{aligned}
& \limsup _{\ell \rightarrow+\infty} a_{\ell}^{-2} \log \mathbb{P}\left(a_{\ell}^{-1}\left|\widetilde{\mathcal{L}}_{\ell, B_{r_{\ell}}}-\widetilde{\mathcal{M}}_{\ell, B_{r_{\ell}}}\right|>\delta\right) \\
& \leq \limsup _{\ell \rightarrow+\infty} a_{\ell}^{-2} \log \frac{a_{\ell}^{-2} \mathbb{E}\left[\left|\widetilde{\mathcal{L}}_{\ell, B_{r_{\ell}}}-\widetilde{\mathcal{M}}_{\ell, B_{r_{\ell}}}\right|^{2}\right]}{\delta^{2}} \\
& =\limsup _{\ell \rightarrow+\infty} a_{\ell}^{-2} \log \mathbb{E}\left[\left|\widetilde{\mathcal{L}}_{\ell, B_{r_{\ell}}}-\widetilde{\mathcal{M}}_{\ell, B_{r_{\ell}}}\right|^{2}\right] .
\end{aligned}
$$

Using (3.11) in (4.11) and taking $a_{\ell}=o\left(\sqrt{\log \log r_{\ell} \ell}\right),(4.10)$ holds.

\section{Acknowledgements}

The authors would like to thank an anonymous referee for his/her valuable comments which improved the quality of the present work.

\section{References}

Baldi, P., Marinucci, D., and Varadarajan, V. S. On the characterization of isotropic Gaussian fields on homogeneous spaces of compact groups. Electron. Comm. Probab., 12, 291-302 (2007). MR2342708.

Benatar, J., Marinucci, D., and Wigman, I. Planck-scale distribution of nodal length of arithmetic random waves. Journal d'Analyse Mathématique, 141 (2), 707-749 (2020). DOI: 10.1007/s11854-020-0114-7.

Bérard, P. Volume des ensembles nodaux des fonctions propres du laplacien. In Séminaire de Théorie Spectrale et Géométrie, Année 1984-1985, pp. IV.1-IV.9. Univ. Grenoble I, Saint-Martin-d'Hères (1985). MR1046051.

Berry, M. V. Regular and irregular semiclassical wavefunctions. J. Phys. A, 10 (12), 2083-2091 (1977). MR489542.

Cammarota, V. Nodal area distribution for arithmetic random waves. Trans. Amer. Math. Soc., 372 (5), 3539-3564 (2019). MR3988618.

Cammarota, V. and Marinucci, D. A quantitative central limit theorem for the Euler-Poincaré characteristic of random spherical eigenfunctions. Ann. Probab., 46 (6), 3188-3228 (2018). MR3857854.

Dembo, A. and Zeitouni, O. Large deviations techniques and applications, volume 38 of Applications of Mathematics (New York). Springer-Verlag, New York, second edition (1998). ISBN 0-387-98406-2. MR1619036.

Döring, H. and Eichelsbacher, P. Moderate deviations via cumulants. J. Theoret. Probab., 26 (2), 360-385 (2013). MR3055808. 
Ledoux, M. A note on large deviations for Wiener chaos. In Séminaire de Probabilités, XXIV, 1988/89, volume 1426 of Lecture Notes in Math., pp. 1-14. Springer, Berlin (1990). MR1071528.

Marinucci, D. and Peccati, G. Random fields on the sphere. Representation, limit theorems and cosmological applications, volume 389 of London Mathematical Society Lecture Note Series. Cambridge University Press, Cambridge (2011). ISBN 978-0-521-17561-6. MR2840154.

Marinucci, D., Peccati, G., Rossi, M., and Wigman, I. Non-universality of nodal length distribution for arithmetic random waves. Geom. Funct. Anal., 26 (3), 926-960 (2016). MR3540457.

Marinucci, D. and Rossi, M. On the Correlation Between Nodal and Nonzero Level Sets for Random Spherical Harmonics. Annales Henri Poincaré (2020). DOI: 10.1007/s00023-020-00985-3.

Marinucci, D., Rossi, M., and Wigman, I. The asymptotic equivalence of the sample trispectrum and the nodal length for random spherical harmonics. Ann. Inst. Henri Poincaré Probab. Stat., 56 (1), 374-390 (2020). MR4058991.

Marinucci, D. and Wigman, I. On nonlinear functionals of random spherical eigenfunctions. Comm. Math. Phys., 327 (3), 849-872 (2014). MR3192051.

Nourdin, I. and Peccati, G. Stein's method on Wiener chaos. Probab. Theory Related Fields, 145 (1-2), 75-118 (2009). MR2520122.

Nourdin, I. and Peccati, G. Normal approximations with Malliavin calculus. From Stein's method to universality, volume 192 of Cambridge Tracts in Mathematics. Cambridge University Press, Cambridge (2012). ISBN 978-1-107-01777-1. MR2962301.

Nourdin, I., Peccati, G., and Rossi, M. Nodal statistics of planar random waves. Comm. Math. Phys., 369 (1), 99-151 (2019). MR3959555.

Nualart, D. and Peccati, G. Central limit theorems for sequences of multiple stochastic integrals. Ann. Probab., 33 (1), 177-193 (2005). MR2118863.

Peccati, G. and Rossi, M. Quantitative limit theorems for local functionals of arithmetic random waves. In Computation and combinatorics in dynamics, stochastics and control, volume 13 of Abel Symp., pp. 659-689. Springer, Cham (2018). MR3967400.

Rudzkis, R., Saulis, L., and Statuljavičus, V. A general lemma on probabilities of large deviations. Litovsk. Mat. Sb., 18 (2), 99-116, 217 (1978). MR0501287.

Schulte, M. and Thäle, C. Cumulants on Wiener chaos: moderate deviations and the fourth moment theorem. J. Funct. Anal., 270 (6), 2223-2248 (2016). MR3460239.

Szegő, G. Orthogonal polynomials. American Mathematical Society, Providence, R.I., fourth edition (1975). American Mathematical Society, Colloquium Publications, Vol. XXIII. MR0372517.

Todino, A. P. A quantitative central limit theorem for the excursion area of random spherical harmonics over subdomains of $\mathbb{S}^{2}$. J. Math. Phys., 60 (2), 023505, 33 (2019). MR3916834.

Todino, A. P. Nodal lengths in shrinking domains for random eigenfunctions on $S^{2}$. Bernoulli, 26 (4), 3081-3110 (2020). MR4140538.

Wigman, I. Fluctuations of the nodal length of random spherical harmonics. Comm. Math. Phys., 298 (3), 787-831 (2010). MR2670928. 
Yau, S. T. Survey on partial differential equations in differential geometry. In Seminar on Differential Geometry, volume 102 of Ann. of Math. Stud., pp. 3-71. Princeton Univ. Press, Princeton, N.J. (1982). MR645729. 